\title{
Social disruption of performance on a DRL schedule'
}

LADD WHEELER AND HANK DAVIS 2,3

NAVAL MEDICAL RESEARCH INSTITUTE AND UNIVERSITY OF MARYLAND

Four rats were trained to stable performance on a schedule which reinforced only bar presses exceeding an inte:-response time of ten sec. (DRL $10 \mathrm{sec}$.). The trained animats, when performing in the presence of other animals, emitted responses having shorter inter-response times than when alone, and as a consequence received fewer reinforcements than when alone. These results are consistent with the theory that being in the presence of other organisms increases arousal, which in turn increases the probability that the dominant response will be emitted.

A recent theory of social facilitation holds that organisms in a group are more aroused than when alone and that this greater arousal increases the probability that the dominant response in the hierarchy will be emitted (Zajonc, 1965). A stated implication of this position is that the presence of other organisms facilitates performance once learning has occurred, because the correct response is then dominant.

In many situations, however, the same response is reinforced at one point in time and not reinforced at another point in time. The presence of other organisms should disrupt performance in such a situation by enhancing the dominant response and thus causing it to occur at times when it will not be reinforced. The research described below demonstrates such social disruption of performance.

\section{Subjects}

Four male Long-Evans rats from the Naval Medical Research Institute colony were obtained at four months of age. Prior to training, the animals were maintained in a group cage with ad lib feeding. The animals were trained during their fifth and sixth months, during which time they were individually caged and maintained at approximately $80 \%$ of free-feeding weight. Training

Training and testing were done in a Skinner box 12 in. $x 12$ in. $x 9$ in. (length $x$ width $x$ height). The feeder delivered $45 \mathrm{mg}$ Noyes pellets. After receiving 100 reinforcements on a continuous reinforcement schedule, the animals were given approximately 40 training sessions of 80 reinforcements each over a two month period on a DRL $10 \mathrm{sec}$, reinforcement schedule (Differential Reinforcement of Low Rates). On a DRL $10 \mathrm{sec}$. schedule a response is reinforced only if it occurs 10 or more sec. after the preceding response. If a response occurs sooner than $10 \mathrm{sec}$. after the preceding response, the timer resets and another $10 \mathrm{sec}$. must elapse before a response will be reinforced.

\section{Testing}

Experimental sessions occurred on four consecutive days at the same time each day. On Days 1 and 2, each animal was given $40 \mathrm{~min}$. alone on the DRL $10 \mathrm{sec}$. schedule. On Days 3 and 4 , each animal was given $40 \mathrm{~min}$. on the DRL $10 \mathrm{sec}$. schedule with another animal in the cage. The naive animal was of the same strain, sex, and approximate age and weight as the DRL animal. A print out counter pulsed once-persec. recorded inter-response times. The animals were monitored via closed circuit television.

\section{Results}

Table 1 presents summary data for each animal alone and paired. The number of inter-response times is shown to indicate the base of the medians and percentages. As is apparent from Table 1, all four animals had a shorter median inter-response time when in the paired situation than when alone $(t=5.08$, $p<.02$ ). Further, every animal had a smaller percentage of his responses reinforced when in the paired situation than when alone $(t=5.96, p<.01)$.

The distributions of inter-response times for each animal are presented in Fig. 1. These distributions clearly indicate that the paired situation resulted in shorter IRTs than did the alone situation.

DRL-1. This animal was the poorest performer when alone. Yet placing him with another animal produced more inter-response times from 2 to $6 \mathrm{sec}$. and fewer from 9 to $14 \mathrm{sec}$. The greater frequency of inter-response times equal to or greater than $30 \mathrm{sec}$. in the paired situation was a result of periods of fighting and postural threat. Fighting on Day 3 led to the naive animal completely leaving the cage after $30 \mathrm{~min}$., squeezing out under the hinged cage lid.

DRL-2. The frequency distribution of inter-response

Table 1. Indices of DRL performance: alone and paired

DRL-1 DRL-2 DRL-3 DRL-4

\begin{tabular}{lllll}
\hline $\begin{array}{l}\text { Number of inter-response times } \\
\quad \text { Alone }\end{array}$ & 653 & 457 & 566 & 463 \\
$\quad \begin{array}{l}\text { Paired } \\
\text { Median inter-response time }\end{array}$ & 370 & 451 & 377 & 472 \\
$\quad$ Alone & 6.7 & 9.0 & 8.4 & 10.5 \\
$\quad \begin{array}{l}\text { Paired } \\
\text { Percent of responses reinforced }\end{array}$ & 5.6 & 7.0 & 7.8 & 10.2 \\
$\quad \begin{array}{l}\text { Alone } \\
\text { Paired }\end{array}$ & 31.6 & 50.7 & 42.5 & 65.6 \\
\hline
\end{tabular}

Note-After applying square root transformations of (Alone-Paired) difference scores, $t$ for median inter-response time $=.08$ (p<.02) and $t$ for percent of responses reinforced $=5.96(p<.01)$. Both tests arc two-tailed with 3 df. 

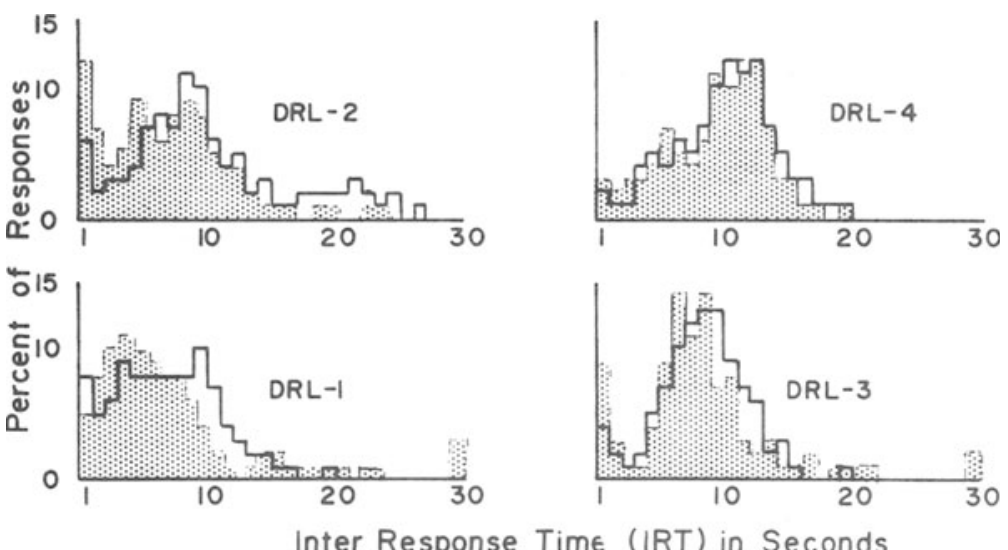

Fig. 1. Distributions of Inter-response times. The heavily outlined white distribution is of IRTs when alone; the spotted distribution is of IRTs when paired

Inter Response Time (IRT) in Seconds with a naive·animal.

times when alone was characterized by a large number in the 20-sec. range and a peaking of responses at 9-10 sec. When paired with another animal, DRL-2 increased the frequency of 1 to $5 \mathrm{sec}$. inter-response times at the expense of inter-response times from 9 to about 25 sec. Due to fighting on Day 4, DRL-2 left the cage after $22 \mathrm{~min}$.

DRL-3. When paired with another animal, this $S$ most strikingly increased inter-response times of 1 sec. and, to a lesser degree, of 6-7 sec. There was also an increase in inter-response times of 17 sec. and greater, due to nonresponding when the naive animal was near the lever and food cup. DRL-3 left the cage after $35 \mathrm{~min}$. on Day 3 after sporadic fighting.

DRL-4. Pairing this animal produced only a slight change in inter-response times, increasing the 1-3 sec. and 6 sec. IRTs.

A discussion of territoriality under schedule conditions similar to these is available elsewhere (Davis \& Wheeler, 1966).

\section{Discussion}

Animals on a DRL reinforcement schedule produced significantly shorter inter-response times in the presence of another animal than when alone and consequently received fewer reinforcements. Shorter inter-response times in the presence of another animal were predicted from Zajonc's theory (1965) that arousal is increased by the presence of other organisms and that the probability of emitting the dominant response is therefore enhanced. While the obtained results were predicted from Zajonc's theory, they are not consistent with his expectation that performance of a well-learned behavior is facilitated by the presence of other organisms. Under conditions such as the DRL schedule provides, when reinforcement of responses is dependent upon the passage of time, performance may be disrupted.

\section{References}

Davis, H., \& Wheeler, L. Social interaction on different schedules of reinforcement. Psychon. Sci., 1966, 4, 389-390.

Zajonc, R. B. Social facilitation. Science, 1965, 149, 269-274.

\section{Notes}

1. From Bureau of Medicine and Surgery, Navy Department, Research Task MF022,01.03-1002. The opinions and statements contained herein are the private ones of the writers and are not to be construed as official or as reflecting the views of the Navy Department or the Naval Service at large.

2. We thank Ira Donenfeld for his skillful assistance.

3. The experiments reported herein were conducted according to the principles enunciated in "Guide for Laboratory Animal Facilities and Care" prepared by the Committee on the Guide for Laboratory Animal Resources, National Academy of Sciences-National Research Council. 